\title{
Analysis of Effect and Mechanism of Different Type Buffer for Reducing Pressure Pulsation
}

\author{
Zhenqian Zhang ${ }^{1, a}$, Yongsheng $\mathrm{Su}^{2, \mathrm{~b}}$ \\ ${ }^{1}$ School of Mechanical and Power Engineering, East China University of Science and Technology, \\ Shanghai 200237, China; \\ ${ }^{2}$ School of Mechanical and Power Engineering, East China University of Science and Technology, \\ Shanghai 200237, China; \\ A18317052165@163.com, byssu@ecust.edu.cn
}

Keywords: pressure pulsation; buffer; flow field analysis; Fluent

\begin{abstract}
The pressure pulsation of network can be reduced effectively by using buffer, discussing the effect of the tangential inlet buffer and radial buffer with different outlet conditions. The reason that affects buffer performance can be gotten through the analysis of internal flow flied and fluid path lines of different buffers. Five groups experiments of different outlet pressure of the compressor are used to provide boundary conditions for Fluent simulation calculation .The result shows that the tangential inlet buffer has better performance than radial inlet buffer for different pressure level.
\end{abstract}

\section{Introduction}

Piston compressor is essential equipment in petrochemical industry,it is mainly because of its simple structure and high discharge pressure.However,the compressor suck and discharge working medium intermittently generating pressure pulsation of the outlet.Exciting force changed with the time will be produced when the pulsating fluid flow through orifice,throttle valve or elbow etc.Network vibration will be generated by the certain frequency exciting force.The vibration not only makes noise pollution but also affects the state of run-time machine,or worse, bring about fatigue failure.In some case, if the exciting frequency is close to the natural frequency of the equipment,resonance will be generated.Moreover,process unit of continuous production require limitations of pressure pulsation,therefore,it is necessary to take measures to decrease and control the pressure pulsation.

There are many measures that can be used to control the pressure pulsation of the pipelines,installing buffer beside the outlet of the compressor is an effective way.The seventies of last century, the former Soviet union experts analyzed the cause of vibration of pipelines systematically and comprehensively,and raised methods to reduce the pressure pulsation[1].Combined with experiment and theory analysis,Xian's Jiaotong university' experts transform sections of pipelines into different transition matrix proceeding from wave propagation,and write programs to calculate pressure pulsation and natural frequency of complex pipelines[2] . In recent years,computational fluid dynamics developed rapidly,study of flow field and effect of buffer used CFD increases gradually.Different buffers have different effect in reducing pressure pulsation,this can be accounted from their inner flow field,and inner structure will affect the property,optimization can be obtained through changing the inner structure parameter[3][4]. Document[5]analyze the property and transmission loss of buffer under different frequencies by CFD.In this paper,proceeding from inner flow field the analysis the mechanism that tangential and radial inlet buffer reducing pulsation is elaborated based on experiment path line,pressure and velocity field of different types of buffer. 


\section{Pressure pulsation standard of pipelines}

Chart.1 Classification standard of Pipelines

\begin{tabular}{|l|c|c|c|}
\hline $\begin{array}{l}\mathrm{P} \quad(\mathrm{kw}) \text { Exit Pressure } \\
(\mathrm{bar})\end{array}$ & $\mathrm{W}<55$ & $55<\mathrm{W}<220$ & $220<\mathrm{W}$ \\
\hline $\mathrm{P}<35$ & 1 & 2 & 2 \\
\hline $35<\mathrm{p}<70$ & 2 & 2 & 3 \\
\hline $70<\mathrm{p}<200$ & 2 & 3 & 3 \\
\hline
\end{tabular}

Pulsating pressure caused by intermittently suction-exhaust manner and peculiarity of vent valve give rise to the vibration of the pipe network connected with the compressor and damage the valve parts and pressure meter. Buffer connected between piston compressor and pipe networks play an effective role in reducing the pressure pulsation of the outlet.The range of pressure pulsation can be evaluated by pressure non-uniformity $\delta$.pressure non-uniformity $\delta$ can be calculated as $\delta=\frac{p_{\max }-p_{\min }}{p_{\text {ave }}}$

American petroleum institute classify the calculation of pipelines pressure pulsation into three kinds. According to API[9], when the pressure is under 3.5bar the allowable pressure pulsation range is calculated as formula 1. Coupled with allowable pressure pulsation for single part,when pressure is between 3.5bar and 350bar,calculation is made by formula 2 .

$$
P_{1}=\frac{4.1}{\left(P_{L}\right)^{\frac{1}{3}}} \% \quad(1) \quad P_{1}=\sqrt{a / 350} \frac{400}{\left(P_{L} \bullet D_{I} \bullet f\right)^{0.5}}
$$

Where $P_{1}$ is peak-to-peak pulsation value and $P_{L}$ is average pressure in bar, and a is the sound speed in $\mathrm{m} / \mathrm{s}, D_{I}$ and $f$ are internal diameter of the pipeline and pulsation frequency measured by

mm and Hz respectively. $f=\frac{N \bullet z}{60}, N$ is the speed of spindle(r/min),z is integer 1,2 , 3...related to natural frequency.

The standard not only gives allowable pressure pulsation range for different pressure level but also proposes the design of buffer reducing pulsation.Buffer can smooth the airflow in the pipelines connected with the piston compressor,the design volume of the buffer should be tenfold volume of the cylinder according to experiment.

\section{Experimental process}

Experimental installation is 11ZA-15/8 vertical duplex single-acting water-cooled air compressor with revolving speed $500 \mathrm{r} / \mathrm{min}$,shaft power $12 \mathrm{kw}$ and exhaust $1.5 \mathrm{~m} 3 / \mathrm{min}$. There are two kinds of buffer tangential inlet and radial inlet with the same structure size.The diameter is 200mm,and length is $800 \mathrm{~mm}, 35 \mathrm{~mm}$ is the diameter of inlet pipe. There are two pressure transducers, one is placed at 6 times diameter away from inlet, the other is placed at 5 times diameter away from the outlet of the buffer. The exhaust pressure is related with the discharge valve and the back-pressure of the pipelines.

Chart. 2 The inlet and outlet pressure

\begin{tabular}{|l|c|l|l|l|l|}
\hline Inletpressure(bar) & 1.5 & 2.5 & 3.5 & 4.5 & 5.5 \\
\hline Outlet pressure(bar) & 1.46 & 2.45 & 3.46 & 4.45 & 5.46 \\
\hline
\end{tabular}

Different exhaust pressure can be achieved through adjusting discharge valve and back-pressure of the pipelines.In this paper five groups inlet and outlet pressure are achieved by changing the back-pressure in order to study the property of the buffer for different pressure levels,in addition,the 
inner flow field of two kinds of buffer are analyzed in comparison.

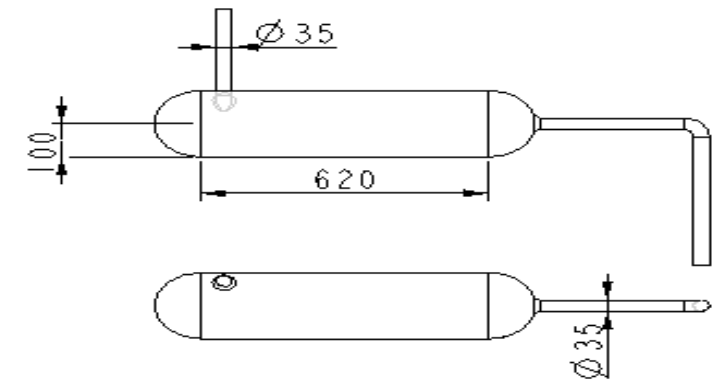

Fig.1Tangential inlet buffer

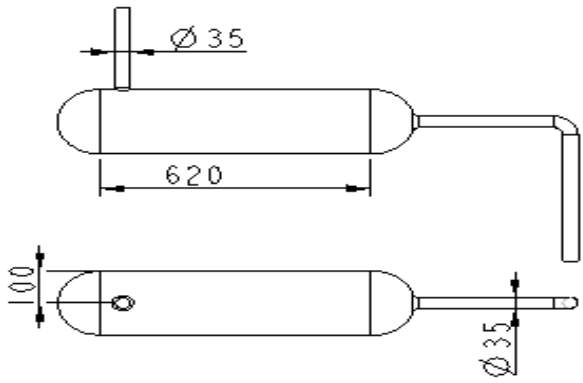

Fig.2 Radial inlet buffer

\section{Numerical modeling}

The buffer model is drew by Pro-E in accordance with experimental buffer and saved as parasolid format so that it can be imported into ICEM directly.the dimension of tangential inlet is shown as figure 1 and the radial inlet buffer size is shown as figure 2 .The model is meshed by unstructured grids,flow medium is ideal compressible gas, $k-\varepsilon$ turbulence model is applied for well adaptation to high Reynolds number,and the smallest residual is 0.0001 .The setting of boundary is the key to model simulation,the ideas that apply changing sine pressure inlet condition through Fluent secondary development using udf programming and constant pressure as outlet condition,domestic and foreign documents mentioned,are roughly same.Xi'an Jiaotong University Song huihui set constant pressure 101325 as outlet condition and $\mathrm{p}=101325[1+0.13 \sin (38.2 t)]$ as the inlet condition when study the effects of orifice for pressure pulsation.

M. Shiddi bqur Rahman set experimental inlet pressure measured by transducer as inlet boundary condition and constant pressure as outlet condition condition when study the outcome of buffer at different frequencies[5].In order to improve calculation precision the initial condition of transient calculation should be accorded with the boundary condition of steady calculation,and the character of discharge valve should be take into account.In view of above cases new adjustment of inlet boundary condition is improved as: $p=p_{0}+\Delta p \cdot \sin \left(\omega t-\frac{\pi}{2}\right), \omega$ is the angular frequency, $\Delta p$ is the amplitude of fluctuating pressure, $p_{0}$ is initial pressure.

\subsection{Steady analysis}

Simulation process has two phases,first,it is the steady analysis of flow field and streamline,after that it is the transient analysis of process of pressure pulsation on the basis of steady analysis. According to the result of steady analysis,figure 3 is the pressure nephogram of the face through center line and figure 4 is the velocity contour.It can be found that the pressure decrease from the wall to the center presenting layer distribution.The velocity contour is similar with the pressure nephogram and the velocity layer close to the wall is thickest,this is related to the flow path.

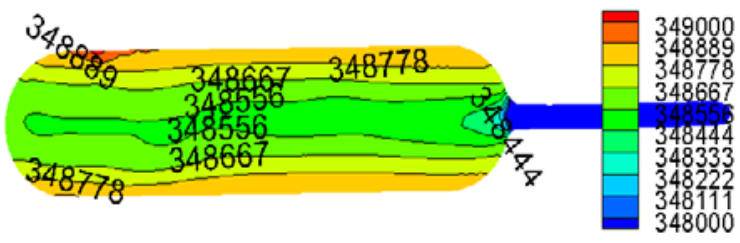

Fig.3 Tangential inlet pressure contour

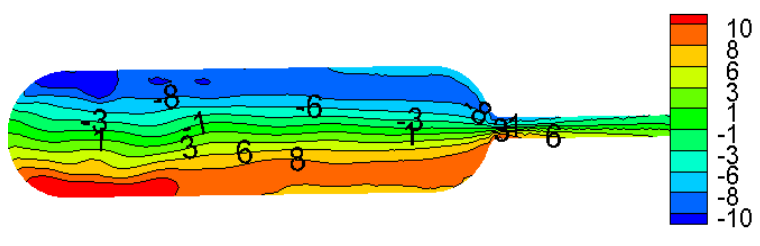

Fig.4 Tangential inlet velocity contour

The path line of flow is presented as figure 5,it can be found that layer distribution of pressure and velocity contour is the result of the rule of flow path line.Fluid medium is divided into two parts after coming into the buffer,one part close to the wall flow towards the outlet in a logarithmic spiral way and constitute the outside of the flow field,the other fill the head near the inlet in a spiral way firstly and then flow towards the outlet in spiral manner,this part constitute the inner part of the flow 
field.The spiral radius of inner part is smaller than the outside part.Compared with the inner flow,the flow of outside has more legible flow path line and low disorder degree.

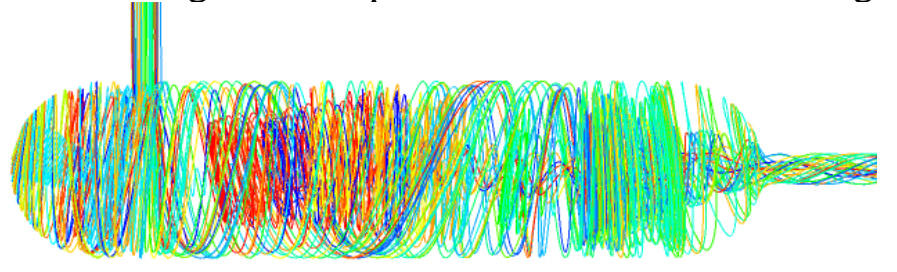

Fig.5 Tangential inlet pathlines

According to the simulation result,the outside flow has the same tangential velocity, the average thickness of outside is $0.015 \mathrm{~m}$ shown as figure 4.If fluid medium travel around same times in the buffer,different radii will lead to different path line length.The revolving laps of the medium change with the pressure difference between inlet pressure and outlet pressure,the larger the pressure difference is the less revolving laps will be.The outside flow can be divided into several control volumes with same thickness ideally,the control volumes have same tangential velocity,there will be relative movement between adjacent control volumes because of different radii,differential pressure contributed to homogenization will be created between adjacent control volumes on the basis of relative movement,homogenization is connected with differential pressure and diffusion time between different fluid particles,large differential pressure and longer diffusion time will be beneficial to homogenization.

Inner flow field of tangential inlet buffer and radial inlet buffer vary considerably in complexity,this is mainly displayed in two aspects. With the same amount of mesh and boundary conditions of inlet and outlet,radial inlet buffer simulation is more difficult to achieve convergent result than the tangential buffer and need more calculation steps and smaller relaxing factor.Besides that,the degree of chaos of pressure and velocity contour of radial inlet buffer is high,it can be found that fluid medium flow towards three directions with no discipline.Different pressure regions cross each other and this is bad for homogenization of buffer flow field. Path line and pressure contour are presented as figure 6 and figure 7 .

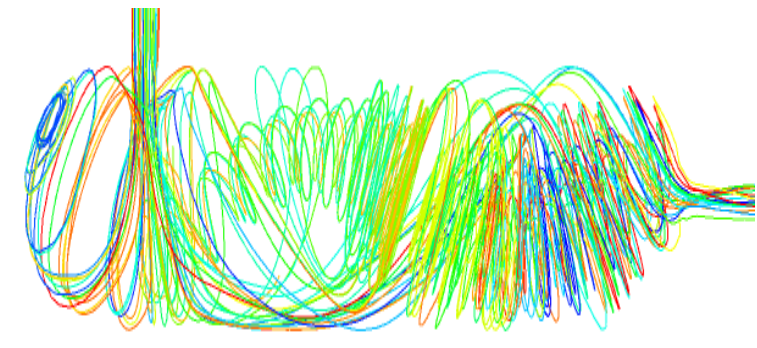

Fig.6 Radial inlet pathlines

\subsection{Transient analysis}

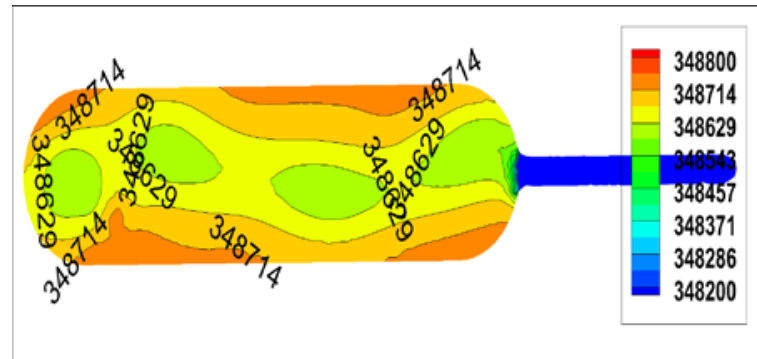

Fig.7 Radial inlet pressure contour

Three groups of comparison analysis between radial inlet buffer and tangential inlet buffer transient simulation is carried out by Fluent based on experimental data with same monitoring position and calculation cycle length.It can be found that the property of tangential buffer is better than that of radial inlet buffer.Chosen datum are 1.5bar, 3.5bar, 5.5bar and pressure pulsating degree are $\delta_{1}=7.3 \%, \delta_{2}=6 \%, \delta_{3}=6 \%$ respectively. Outlet pressure detection is carried out during transient calculation with $0.001 \mathrm{~s}$ interval,and the detection datum is ploted as follows. 


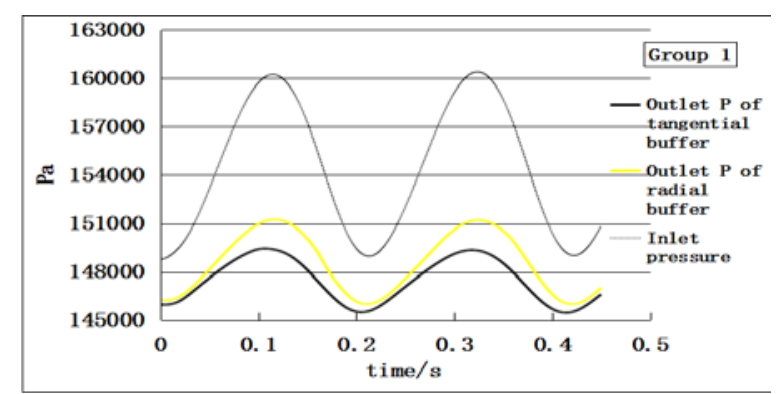

Fig.8 Inlet and outlet pressure of group 1

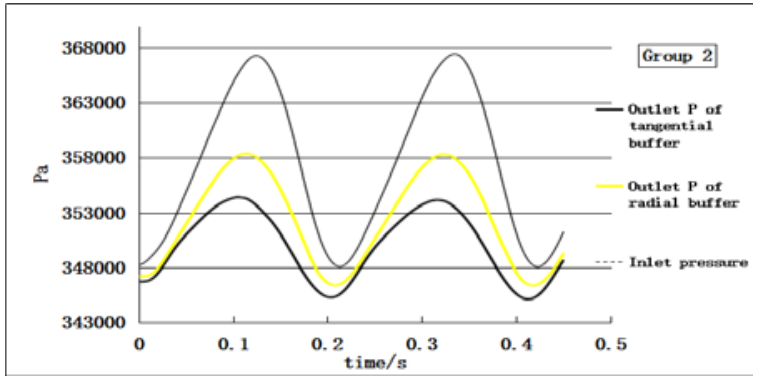

Fig.9 Inlet and outlet pressure of group 2

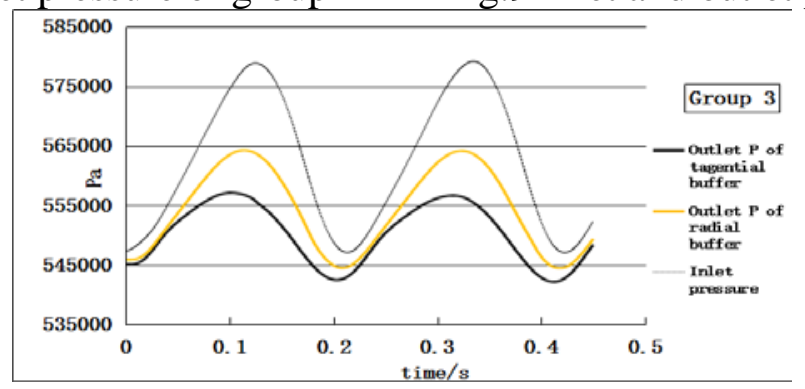

Fig.10 Inlet and outlet pressure of group 3

The transient results of three differeent pressure groups can be calculated with detection datum. The results of tangential buffer are $\delta_{1 q}=2.66 \%, \delta_{2 q}=2.5 \%$,

$\delta_{3 q}=2.54 \%$ repectively with different pressure groups. The pressure pulsation results of radial buffer are $\delta_{1 J}=3.4 \%, \delta_{2 J}=3.5 \%, \delta_{3 J}=3.63 \%$.

Chart.3 Inlet and outlet pressure pulsation

\begin{tabular}{|c|l|l|l|}
\hline $\begin{array}{l}\text { Group } \\
\text { Range }\end{array}$ & $\begin{array}{l}\text { Inlet pressure } \\
\text { pulsation }\end{array}$ & $\begin{array}{l}\text { Tangential exit pressure } \\
\text { pulsation }\end{array}$ & $\begin{array}{l}\text { Radial exit Pressure } \\
\text { pulsation }\end{array}$ \\
\hline 1 & $7.3 \%$ & $2.66 \%$ & $3.4 \%$ \\
\hline 2 & $6 \%$ & $2.5 \%$ & $3.5 \%$ \\
\hline 3 & $6 \%$ & $2.54 \%$ & $3.63 \%$ \\
\hline
\end{tabular}

It is observed that the effects of tangental and radial buffer are both stable with different pressure levels.In addition, the performance of tangential inlet buffer is better than that of radial inlet buffer. The allowed pressure pulsation are $3.49 \%, 2.8 \%, 11 \%$ in sequence with three different pressure levels according to the standard of API 168. It is calculated that the pressure pulsation passed the tangential buffer is up to the standard.

\section{Summary}

The following conclusions can be obtained through above analysis

(1)The inner flow field of radial inlet buffer is more complex than the flow field of tangential inlet buffer,but the pressure mixing degree of radial inlet buffer is inferior to the mixing degree of tangential inlet buffer.

(2)At the same structure size, the tangential inlet buffer has better property in reducing pressure pulsation than radial inlet buffer.

(3)The buffer has stable property in reducing pressure pulsation for different pressure levels.

\section{References}

[1]M Shiddiqur R. Experimental and Numerical Study of Snuber in Hydrogen Compressor[J]. International Journal of Science and Engineering, 2012, 3(2): 21-25.

[2]Jeong H M, Chung H S, Akbar W A, et al. Numerical analysis on various models of pressure snubbers in the hydrogen gas compressing system[J]. Journal of Mechanical Science and 
Technology, 2008, 22(4): 761-769.

[3]He S, Jiao Z, Guan C, et al. Research on pulsation attenuation characteristics of expansion chamber in hydraulic systems[C]//Industrial Informatics (INDIN), 2012 10th IEEE International Conference on. IEEE, 2012: 735-739.

[4]Broerman E L, Smolik M A, Scrivner C M. Helmholtz Absorbers: Experiments in Controlling Resonant Pulsation Without the Use of Orifice Plates[C]//ASME 2007 Pressure Vessels and Piping Conference. American Society of Mechanical Engineers, 2007: 449-456.

[5]Standard A P I. 618[J]. RECIPROCATING COMPRESSORS FOR PETROLEUM, CHEMICAL, AND GAS INDUSTRY SERVICES, 4th Edition, American Petroleum Institute, Washington, DC, 1995.

[6]Rahman M S, Lee G H, Utomo T S, et al. A Study on Gas Pressure Fluctuation Characteristics inside the Snubber and Pipe of Hydrogen[J]. Advances in Mechanical Engineering, 2008, 2009. 